\title{
Students' epistemological beliefs from two studies of calculus and physics
}

\author{
Anna McLean Phillips ${ }^{1}$ and Caroline J. Merighi ${ }^{2}$ \\ ${ }^{1}$ Department of Physics \& Astronomy, Tufts University, 574 Boston Avenue, Medford, MA, 02155 \\ ${ }^{2}$ Department of Education, Tufts University, 12 Upper Campus Road, Medford, MA, 02155
}

\begin{abstract}
Students often take introductory physics concurrently with calculus, and the two disciplines are deeply intertwined. While students' epistemological beliefs in physics have been studied extensively, less work has examined their beliefs in calculus. Even less is known about how students' epistemological beliefs in physics and calculus may relate, interact, and shift together or separately. We report on results of two independent studies: one including interviews of calculus students and one of introductory physics students. Across both studies, we found that students made statements consistent with an algorithmic view of calculus while also expressing a view of physics as about sense-making. Through close analysis of one physics student, we also show that it is possible for a student to shift from a novice-like view of physics to an expert-like one while maintaining a algorithmic view of calculus.
\end{abstract}

Keywords: epistemology, introductory physics, introductory calculus

\section{INTRODUCTION}

Students' beliefs about physics, including their beliefs about the role of mathematics in physics, have been studied in depth for quite some time (e.g., [1, 2]). These beliefs are often an explicit target of instruction, both as a goal in and of themselves, and because of work strongly suggesting that students who view physics as a coherent system of ideas learn the core ideas of introductory physics more successfully than those who view physics as a set of facts and formulas [2]-4].

There are comparatively few studies of students' epistemologies in mathematics as they relate specifically to introductory calculus [5], though it is generally recognized that beliefs can form an important part of the context for learning [6]. Studies have documented that students often enter college with mathematical knowledge that consists mostly of memorized procedures and they believe that mathematics is a collection of rules and facts to be remembered [7].

While studies have examined students' use and understanding of mathematics within physics courses (e.g., [8]), less attention has been paid to how students' epistemological beliefs in physics and calculus relate or interact. For physics and engineering students, introductory physics relies on and uses calculus heavily. Therefore, one might wonder if students' beliefs about calculus may impact their experience within physics courses or vice versa. There is ample evidence that physics courses may influence students' beliefs about physics [9]. Newer work shows that courses that shape students' beliefs about what it means to do and learn physics may also influence their beliefs and learning in subsequent courses [10]. This would support the possibility that students' epistemologies in calculus and physics may be related and shift together.

On the other hand, students' epistemological beliefs in physics are dynamic and dependent on context [11], and the same may well hold true for students' views of calculus. That would suggest that students' beliefs about what it means to do calculus and what counts as mathematical knowledge may be different within the contexts of mathematics and physics courses. Furthermore, it may be possible that students de- velop more sophisticated views of calculus without similar development of their views of physics and vice versa.

We present preliminary results from two independent studies involving interviews with undergraduate students in introductory physics and calculus. Study A was designed to examine how a reformed introductory physics class shaped students' epistemological beliefs in physics, and Study B examined students' conceptual understandings of calculus. While neither study sought to uncover how students' epistemologies in calculus and physics were related, participants in both studies spontaneously made statements that explicitly contrasted their views of calculus and physics.

Analysis of interviews from both studies reveal that students' epistemologies of calculus and physics differ substantially. Students who profess a view of physics as a sensemaking process may express that calculus consists of algorithms and procedures, regardless of whether the interview was about physics or calculus. Through Study A, in which students were repeatedly interviewed, we see that students may make progress towards more expert-like beliefs about physics while maintaining algorithmic views of calculus.

\section{METHODOLOGY}

Study A. The goal of study A was to examine how students' beliefs about physics changed while enrolled in a reformed physics course. The course was taught in a similar format to that described in Ref. [12]; class discussions, homework assignments, and laboratories were explicitly designed to shape students' epistemological beliefs in physics. The course was a calculus-based mechanics course, and most of the 50 students enrolled were freshman engineering students. Further information about the course can be found in Ref. [13].

Interview participants were selected based on their responses to the Colorado Learning Attitudes About Science Survey (CLASS) [14] and their first homework assignment. The selection criteria was designed to capture students who would possibly shift their beliefs during the semester; initially students' CLASS responses reflected a novice-like view of physics and whose homework reflected few sense-making 
practices were invited to participate. Only nine such students were found 1 and three agreed to participate. The selection criteria was broadened to include students with novice-like responses on CLASS or homework, and two more students were recruited.

Each student was interviewed four times throughout the semester. The interviews were loosely structured. The interviewer, Phillips, began each session by asking "How are things going in physics?" and would ask questions about students' experiences with lecture, lab, and homework assignments. The interviewer, would ask follow up questions to pursue statements that students had made that were unclear or vague. Each student was asked specifically to compare their experience in physics to their experiences in other classes.

Study B. Participants in Study B were enrolled in a traditional Calculus I course at the same institution as Study A, though the two studies were designed and conducted independently. All 40 students from one section of Calculus I were invited to participate in a single one-on-one interview with Merighi at the end of the semester. Nine students chose to participate; six were first-year students and three were second-year students. There was no overlap in interview participants between the two studies, and no interview participants in Study B were enrolled in the course in Study A.

The interviews were semi-structured and designed around three mathematics tasks that were chosen to gather data on how students think about rates of change and accumulation. The original intent of the tasks was to uncover students' conceptual ideas, thus none of the tasks explicitly asked students about their beliefs about mathematics. However, the ways in which students approached the mathematical tasks and their verbal commentary throughout the interviews gives strong clues about how they view mathematics. In addition, several students spontaneously commented on their experiences in calculus courses and compared it to other courses, revealing both directly and indirectly some of their views about both mathematics and physics.

We examined interviews from both studies to identify moments where students mentioned both calculus (or mathematics more generally) and physics. We preformed a descriptive analysis of these moments to identify evidence of students' beliefs about physics and calculus.

\section{FINDINGS}

\section{A. Across both studies, students expressed contrasting epistemological views in calculus and physics}

Across both studies, participants often framed calculus as consisting of calculations or algorithms while describing physics as requiring sense-making.

In Study B, six of the nine students spontaneously expressed the idea that there is a significant difference between

\footnotetext{
${ }^{1}$ See Ref. [13] for a discussion of this discrepancy between CLASS responses and homework.
}

physics and calculus. Such comments tended to arise in response to the tasks. For example, students were asked to interpret information about the relative position and velocities of two cars given graphs of the cars' velocities. Though this question was designed to evaluate students' understanding of rates of change, it prompted students to contrast what they viewed as doing physics versus doing calculus.

Several students indicated that they could not really use mathematics to help them since an equation was not present. For example, Nate ${ }^{2}$ offered,

One of the things that I just like, like about calculus as opposed to, like, physics, is... I think of this more as physics than calculus because calculus... I'm just so ingrained in like an $\mathrm{x}$ and a $\mathrm{y}$, and a derivative, and an integral, and an area, and like when I have to actually apply it to something like this which is like a pretty real life situation, [it's much more difficult].

Nate describes his experience of calculus is "ingrained" in "an $\mathrm{x}$ and $\mathrm{a} y, "$ and he implies he associates reasoning without equations or numbers as physics rather than calculus.

In Study A, four of five students made statements contrasting physics and math without prompting in interviews 1 and 2. To explore this theme, Phillips began asking students directly about their experiences in mathematics courses in interviews 3 and 4. Three of the five students, Chandra, Sasha, and Kallie were concurrently enrolled in Calculus II. They each made statements about finding mathematics to be more certain, stating that they knew what steps to take in math to solve problems. They expressed that physics required additional thinking and effort. At times, students expressed this as a reason why they were better able to do math. At other times, they expressed why it made physics a more enjoyable experience. For example, near the end of the course, Sasha said,

In math we're literally just given an algorithm to follow and we have to just keep doing the same thing again and again. [...] I can solve random problems, like actual physics problems. [...] It's out in the world and if you tweak the problem this way then what happens? It's not just one type of thing.

The students' statements contrasting mathematics and physics seemed closely tied to their experiences in the two courses; their traditional calculus course emphasized computation while their reformed physics course emphasized physics as a sense-making process.

Two students in Study A, Serena and John, did not express contrasting epistemological beliefs in physics and calculus. Neither was concurrently enrolled in a mathematics course.

\footnotetext{
${ }^{2}$ All names are pseudonyms.
} 
Serena expressed stable views of both physics and mathematics as facts and procedures. She contrasted her experience in the physics course (which she found frustrating), and her past experiences in calculus courses (which she found satisfying). John, however, displayed shifts in his views of physics and, when asked, linked those shifts to his views of mathematics. In the final interview, he reflected on his approach of memorizing all of the proofs in his calculus textbook. He recounted that, at the time, "I felt like there wasn't a different way to go about it." Yet at the end of physics class, he thought that there must be "a million different things to show that that theorem was true with what we had learned prior." His response may have reflected the interview context, where he was talking about sense-making, and may not be reflective of to what he would actually do in future math classes. John was the only student in Study A to suggest that his experience in physics had shaped how he thought about mathematics.

\section{B. Students may experience epistemological shifts in physics while maintaining stable views of calculus}

Four of five students in Study A showed shifting epistemological beliefs in physics. One student, Chandra, frequently contrasted physics and mathematics unprompted by the interviewer. Over the course of the four interviews, her views of physics shifted substantially. Yet her algorithmic view of calculus appeared stable throughout the study. We focus on her trajectory to show how changes in epistemological beliefs in physics need not be accompanied by similar shifts in calculus.

In her first interview, Chandra expressed a novice-like view of physics as facts and formulas:

I don't really trust my intuition in science. Like I rather go with the equations, I'd rather go with like the facts. I'd rather go with the principles behind certain things. (Interview 1)

Chandra appears to view her intuition and everyday experience as untrustworthy as well as distinct from and less useful than facts and equations. While her use of the word "principle" could reflect a more expert-like view of physics, further probing revealed that her use of "principle" appeared to mean physical laws that are often expressed as equations (such as Newton's Second Law).

As the semester progressed, she noted that the physics homework was harder and required a great deal of her time and energy. Unprompted, she contrasted this to her mathematics homework, noting that she could do that work even when quite tired:

[After sports practice] I could do homework, and I would do, like-but I would do, like, math homework or Spanish homework and not what I really need to like use my brain. (Interview 2)

When asked to elaborate, she said,

And for me, the way I do math is, like, I know when I know how to do it. Like, I'll know that, like, okay on this one I can now do any problem, like, related to this topic, and I'll be fine with it. [...] It's strange because I'm like very confident with calculus, but then with physics, it's, like, iffy. (Interview 2)

Chandra's view that she knows when she knows how to do mathematics is consistent with an algorithmic view: once she can do one problem, she can do any on the same topic. For her, physics is different and uncomfortable. At this point in the study, it was unclear if these views were of the two different courses or of the two different disciplines.

In the third interview, Chandra was asked to share about how the physics course was going. She replied,

It's hard but it's good. It's fun to think. I don't know. It would be sucky if you just knew how to do everything. It would be boring. (Interview 3)

Chandra had come to view problem solving and thinking through ideas in physics as "fun," rather than uncomfortable.

In the last interview, Chandra described the differences in the homework between math and physics and made explicit statements about the differences between the disciplines:

I think physics is a lot more, like, interactive homework. Like, you go to office hours, sometimes you just [use the on-line discussion], whereas math you kind of just know it and you just finish it off. Yes, I think that's the difference. But I also think that's the difference between in, like, a science and, like, a math. At least, a math level where I am, like, it hasn't reached the point where you have to, like-I don't know, you either know it or you don't. Whereas, like, science, there's so many ways of getting about it. (Interview 4)

While Chandra allows for the fact that mathematics at a higher level may be different, she offers a clear view of calculus as consisting of one approach, something that she either knows or does not know, without tools for figuring out how to solve things on her own. She contrasts this with physics and science more generally, stating that science has "many ways of getting about it." When asked to elaborate, she said,

If you don't know how to integrate, you're not going to be able to do the homework. Go figure out how you're going to integrate. Whereas physics, if you don't understand torque, you can read about it, you can try to think about it, but you can also think about it in, like, different ways. You can think about it, like, of the momentum, of the motion, of like, the force, like, $\mathrm{F}$ equals MA. So there are, like, different ways of doing it. Whereas if you get a math question, take the inte-like integrate this, and you don't know how to integrate it, [pause, shrug] what are you going to do about it? (Interview 4) 
By the end of the course, Chandra had come to view physics as an interconnected set of ideas, which was tied to her experience of being able to think through how to solve physics on her own. This reflected a shift in her epistemological beliefs about physics. Yet her view of calculus was very much one where approaches were either known or not known, and knowledge had to be looked up, rather than figured out.

\section{DISCUSSION AND CONCLUSION}

Taken together, Study A and Study B provide evidence that students may have unsophisticated or algorithmic views of calculus even if they view physics as involving sensemaking. Further work studying students' epistemologies in calculus and how they relate to their epistemologies in physics could significantly inform research and instruction, particularly given how many students take the courses concurrently.

A limitation of our work is that our interviews only provide evidence of students' professed epistemological beliefs, rather than their enacted beliefs. A close analysis of students' homework assignments or videos of these students working on both mathematics and physics might tell a different story than interviews. It is important to note that this is emergent work looking at students' spontaneous comparisons between physics and calculus, rather than a study specifically designed to compare and contrast beliefs about calculus and physics.

Despite these limitations, our analyses suggest that researchers should be cautious about assuming too many similarities between students' epistemologies in physics and calculus, since we can see shifts in students from more algorithmic and procedural views to more sense-making orientations in physics while simultaneously maintaining strong algorithmic views of mathematics. Further work, including studies specifically designed to probe how students' beliefs about calculus and physics may relate, is clearly needed. In particular, it may be interesting to compare students who are concurrently enrolled in reformed physics and reformed calculus courses with those concurrently enrolled in one reformed and one traditional course.

Our data suggest that impacts of reformed physics courses may be limited by what is happening for students in tradi- tional calculus courses. If one goal of introductory physics courses is for students to make sense of physical phenomena and to use mathematics as a tool to support that, it may be challenging when they hold views of mathematics as merely applying algorithms to find "correct answers" rather than making sense of the underlying principles and meanings of these procedures.

We caution against interpreting these results to mean that students' epistemological beliefs of physics do not "transfer" to calculus and vice versa. Rather, we see our results as consistent with research that epistemologies are fluid and context-dependent [11], and that students may activate different cognitive resources in different situations [15]. The question then is not "How can we get students to use their beliefs about physics to make sense of mathematics?," which could suggest providing more calculus questions grounded in physical scenarios. Rather we suggest considering "How can we support students' in activating similar resources to make sense in both disciplines?" That may involve more explicit coordination between calculus and physics instructors and alignment of curricula which would prove difficult or impossible in traditional university settings. As physics instructors, we can certainly engage our students in conversations about how we, as physicists, use mathematics to make meaning and not only execute algorithms.

Finally, we do not mean to imply that epistemological resources and beliefs that are useful for learning physics are uniformly useful for learning calculus or vice versa. There are meaningful differences between the nature of knowledge in mathematics and physics that should not be ignored, and physicists and mathematicians make meaning with mathematics in different ways [16]. We all agree, however, that we wish students to see mathematics, including calculus, as having meaning and not merely as a set of algorithms.

\section{ACKNOWLEDGMENTS}

We would like to thank David Hammer and the Calculus I instructors at Tufts for facilitating data collection in their courses. We would also like to thank Jessica Watkins for her valuable feedback.
[1] A. DiSessa, Cognition \& Instruction 10, 2 \& 3 (1993).

[2] D. Hammer, Cognition \& Instruction, 12 (1994).

[3] S. Carey \& C. Smith, Educ. Psychologist, 28 (1993).

[4] D. Hammer \& A. Elby, J. of the Learning Sci. 12, 1 (2003).

[5] K. Muis, Rev. of Educ. Research, 74, 3 (2004).

[6] A. Schoenfeld, Mathematical Problem Solving, (1983).

[7] L. Richland, J. Stigler, \& K. Holyoak, Educ. Psychologist, 47, 3 (2012).

[8] S. Rebello et al, In Learning to solve complex scientific problems, edited by D. H. Jonassen. (2007). Routledge.

[9] A. Madsen, S. B. McKagan, \& E. C. Sayre, Phys. Rev. ST Phys. Educ. Res. 11, 010115 (2015).
[10] W. Potter et al, A. J. P., 82, 2, (2014).

[11] A. Gupta, D. Hammer, \& E. R. Redish, J. of the Learning Sci., 19, 3, (2010).

[12] E. F. Redish \& D. Hammer, Am. J. Phys., 77, 7 (2009).

[13] G. Rowe \& A. M. Phillips, PERC Proc. (2016).

[14] W. Adams et al, Phys. Rev. ST Phys. Educ. Res. 2, 010101 (2006).

[15] D. Hammer et al, In Transfer of Learning from a Modern Multidisciplinary Perspective, edited by J. P. Mestre. (2005).

[16] E. F. Redish \& E. Kuo, Sci. \& Educ. 24, 5 (2015) 